\title{
AN OPEN IT INFRASTRUCTURE FOR GREEN TOURISM MANAGEMENT AND PROMOTION: THE INSUBRI.PARKS PROJECT
}

\author{
D. Oxoli ${ }^{1 *}$, V. Terza ${ }^{2}$, M. Cannata ${ }^{3}$, M. A. Brovelli ${ }^{1}$ \\ ${ }^{1}$ Department of Civil and Environmental Engineering, Politecnico di Milano, Como Campus, Como, Italy - \\ (maria.brovelli, daniele.oxoli)@polimi.it \\ ${ }^{2}$ Parco Regionale Spina Verde, Como, Italy - vittorioterza@ spinaverde.it \\ ${ }^{3}$ IST-SUPSI, Institute of Earth Sciences, Canobbio, Switzerland - massimiliano.cannata@ supsi.ch
}

Commission IV, WG IV/4

KEY WORDS: Natural Heritage, E-Tourism, Free and Open Source Software, Geographic Information Systems, Cross-Border Cooperation

\begin{abstract}
:
The design and implementation of modern tourism management systems have to interface with the growing technological attitude of users which nowadays mostly approach the selection of the desired destinations through the web. In view of the above, the local tourism actors have to fully embrace the digital transformation by integrating Information Technologies into their operational routines. With this in mind, it is here presented the development of a technological infrastructure to provide with new digital assets to the natural parks of the Insubria, cross-border region stretching between Northern Italy and Southern Switzerland. This work is carried out within the INSUBRI.PARKS project, funded by the Interreg program of the European Union. The final goal of the project is to increase tourism attractiveness of the Insubria region through the provision of new infrastructure, the design of new tourism experiences, and the deployment of Information Technology tools to support the cross-border tourism management and marketing. The tools development is described in detail and the application prototypes are presented. The work leverages exclusively Free and Open Source Software that provides the infrastructure with large rooms of improvements and customization while reducing both development and maintenance costs. Future directions for the work are outlined by considering the amelioration of the presented tools, the development of additional components, and the practical applications of the infrastructure functionalities from the perspective of both park managers and visitors.
\end{abstract}

\section{INTRODUCTION}

The management of tourism in protected natural areas represents a challenging task since the promotional strategies - which are necessary to unpin the tourism potential of these sites - have to constantly interface with the sustainable exploitation of their landscape and environmental resources to preserve their heritage. Furthermore, this task is often hindered by fragmented political contexts - both local and national - to which each protected area may be subjected. This may prevent optimal management due to competitive actions as well as weak cooperation among the decision-makers (Prell et al., 2009).

In view of the above, we present here the INSUBRI.PARKS project, funded by the Interreg Co-operation Programme 2014 - 2020 (ID 605472) of the European Union (https://interregitaliasvizzera.eu). The project aims at the harmonisation of management and promotion practices through the development of an integrated tourism management system for the natural parks belonging to the Insubria Region, situated on the border between Italy and Switzerland. The Interreg program is an initiative of the European Union aiming at promoting investments to boost cooperation across European regional and local governments in strategic sectors - such as tourism - and to ensure sustainable impacts for people and territories. The project activities encompass multiple actions including the provision of physical infrastructures for pedestrian mobility, the establishment of unified territorial marketing strategies and the develop-

\footnotetext{
${ }^{*}$ Corresponding author
}

ment of a standardized Information Technology (IT) infrastructure to improve information sharing and consuming between project partners and stakeholders.

The present work focuses on the development of the IT infrastructure to support cross-border tourism management and territorial marketing operations (Gretzel et al., 2015, Antonovic et al., 2015, Li et al., 2017). The main goal of the IT infrastructure is to empower cross-cutting management and promotion strategies with a dedicated suite of IT tools. The whole system is implemented by leveraging cutting-edge Free and Open Source Software (FOSS) - both web and GIS (Geographic Information Systems) - by ensuring equitable access to the system to each partner that - in this way - is not bounded by any license or purchase constraint (Brovelli et al., 2017). In general terms, the implementation of smart IT tools based on modern web technologies is key to aid coordination across the organizational boundaries, by reducing technological barriers and costs of decision making that are relevant to geographically distributed organizations (Buhalis, 2003, Chon, 2013, Masron et al., 2016). In this context, GIS play a critical rule since the combination of multimedia content and web technology with GIS provided - in a number of cases - with substantial enhancement to the attractiveness also of tourism systems (Wei, 2012). Moreover, most of the target data have a strong geographical trait that suggests the use of Geographic Information Systems (GIS) technologies as the most logical pattern for the development of the IT infrastructure.

The IT infrastructure is designed to supply functionalities that 
have been outlined by the project's partners as critical to their operational routines as well as to foster the visitors' virtual exploration of the territories. These mainly include the development of a unified spatial database to serve the creation of the project WebGIS platform. In addition, social media (e.g. Twitter) and sensor observations of visitors' counts - collected by a dedicated network that will be deployed within the project are being considered to monitor and analyse the tourism flows in the park areas. This latter information is made accessible to the project's partners by means of an analytical web dashboard that is part of the project IT infrastructure. The preliminary system design was presented in a previous paper by the authors (Oxoli et al., 2019). Indeed, the current paper describes the development work of the IT tools prototypes and provides updates on the project status. The underlying benefits deriving from the co-creation of best management practices by means of open and shared software platform are also discussed alongside the future directions of the work.

The paper continues as follows. Section 2 includes an overview of the INSUBRI.PARKS project. In Section 3, the IT infrastructure architecture is presented. Section 4 reports the description of the main components of the infrastructure. Future directions of the work and conclusions are reported in Section 5.

\section{THE INSUBRI.PARKS PROJECT}

The INSUBRI.PARKS project is funded by the Interreg transnational cooperation program between Italy and Switzerland 2014-2020. The project's partnership is primarily composed of some of the natural parks belonging to the Insubria Region (Figure 1), from which the project inherits its name. The Insubria is a historical-geographical region stretching between the Canton Ticino (Southern Switzerland) and the Lombardy Region (Northern Italy). This region encompasses a number of interconnected landscapes ranging from the plain to the Alpine environment, crossing the region of sub-alpine lakes, such as the Como Lake, and including many densely populated urban centres (Oxoli et al., 2019). The natural parks involved in the project are Spina Verde (https://www.spinaverde.it), Campo dei Fiori (http://www.parcocampodeifiori.it), and Pineta (http://www.parcopineta.org) for the Italian side. For the Swiss side, two parks are involved namely Gole della Breggia (https://tinyurl.com/y243ryft) and Bosco del Penz managed by the municipality of Chiasso (https://www.chiasso.ch) (Figure 1).

The INSUBRI.PARKS project aims at designing and implementing shared tourism strategies and practices to integrate the common natural, cultural and historical heritage characterizing the project area and - at the same time - to leverage the local peculiarities of each natural park and surrounding territories. In support of the co-creation of a shared tourism management system, the provision of new physical infrastructure for pedestrian mobility and the development of a standardized IT infrastructure - empowering territorial promotion and strategic information collection - are considered (see: https://tinyurl.com/rleavcb). To that end, the project's partnership also comprises the non-profit organization Pro Val Mulini ONLUS (http://www.provalmulini.eu), to deal with the historical and cultural aspects of the INSUBRI.PARKS, and the Swiss Regional Tourism Office (OTR) Mendrisiotto Turismo (https://www.mendrisiottoturismo.ch) which provides the know-how on best tourism management practices. Finally, two scientific partners are included. These are the Politecnico di Milano (https://www.polimi.it) from the Italian side, and

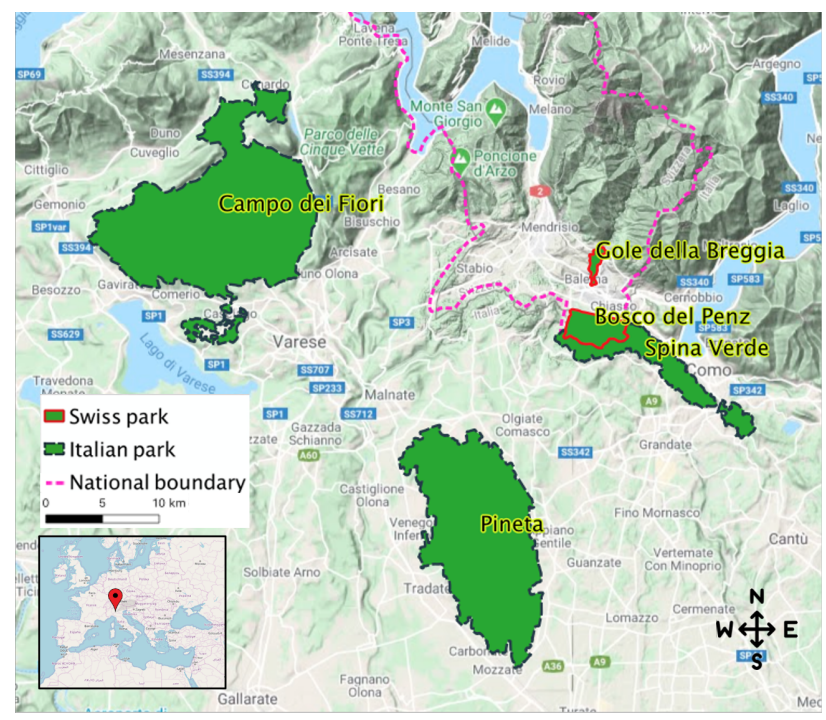

Figure 1. The Insubria region and the natural parks involved in the INSUBRI.PARKS project (basemap: (C)2020 Google)

the Scuola Universitaria Professionale della Svizzera Italiana (SUPSI, http://www.supsi.ch) from the Swiss side which are in charge of the IT infrastructure development.

\section{OPEN IT INFRASTRUCTURE FOR GREEN TOURISM PROMOTION}

Most of the existing studies in the literature suggested the success of many tourism systems being linked to the availability of innovative IT tooling to support both planning and marketing operations on the territories (Jovanović, 2016, Buhalis, Amaranggana, 2015). To that end, the INSUBRI.PARKS project recognizes the adoption of cutting-edge technologies as a key point to accomplish its goals. With this in mind, the main purpose of the IT infrastructure is to empower all the cross-cutting management and promotion operations - involving data - with dedicated IT tools. These operations include the tourism fluxes monitoring and analysis and the virtual exploration of the park attractions, among others (Oxoli et al., 2019).

To achieve the above, a dedicated client-server architecture for the IT infrastructure was designed as follows (Figure 2). The primary component is represented by the data. The different applications of the IT infrastructure rely on a single data endpoint in the back-end which is composed of a PostgreSQL instance (https://www.postgresql.org), one of the most popular FOSS relational database. The database instance is enriched with the spatial extensions PostGIS (http://postgis.net) and pgRouting (http://pgrouting.org), providing support to spatial data storage and manipulation. The database is design to include both digital cartographic data as well as social media and sensors data (see Section 4.1) that are exploited respectively by the WebGIS and the web dashboard client applications. The server side is also based on FOSS and mainly consist of a GeoServer instance (http://geoserver.org) which enables spatial data access and sharing with the client by means of Open Geospatial Consortium standards (OGC, https://www.ogc.org). In addition, istSOS (http://istsos.org) is included to handle sensors observations (see Section 4.2).

The client-side includes two main components that are represented by the WebGIS and web dashboard applica- 
tions. Both components are base on the Python Django (https://www.djangoproject.com) web framework that allows interactions with the back-end components and services outlined above. Considering the web dashboard, this application provides park managers and their collaborators with visualization and exploration tools - namely interactive graphs e summary statistics - for the social media data and sensors observations collected in the project database (see Section 4.4). The web dashboard exploits the D3.js (https://d3js.org) JavaScript library to implement interactive data visualizations. The node.js (https://nodejs.org) JavaScript runtime environment is used upon the Django framework to handle the D3.js functionalities. Finally, the WebGIS application is designed to provide users with access to digital cartographic information of the project areas through interactive map-based functionalities. The digital cartographic information - stored into the PostgreSQLPostGIS database instance - is access by the WebGIS using OGC Standard such as the Web Mapping Service (WMS, https://www.ogc.org/standards/wms) and the Web Feature Service (WFS, https://www.ogc.org/standards/wfs) which are implemented by the GeoServer in the back-end. The WebGIS interface is mainly based on the Openlayers JavaScript library (https://openlayers.org). Enhanced interaction with the information layers is enabled by means of WebGIS plugins such as the Tourist Experience Planner tool. This plugin allows users getting custom suggestions on the most suitable park itineraries according to their expressed preferences on e.g. point of interests or attraction within the parks. The plugin is developed in Python and exploits the pgRouting functionalities in the back-end for custom itineraries computation (see Section 4.3).

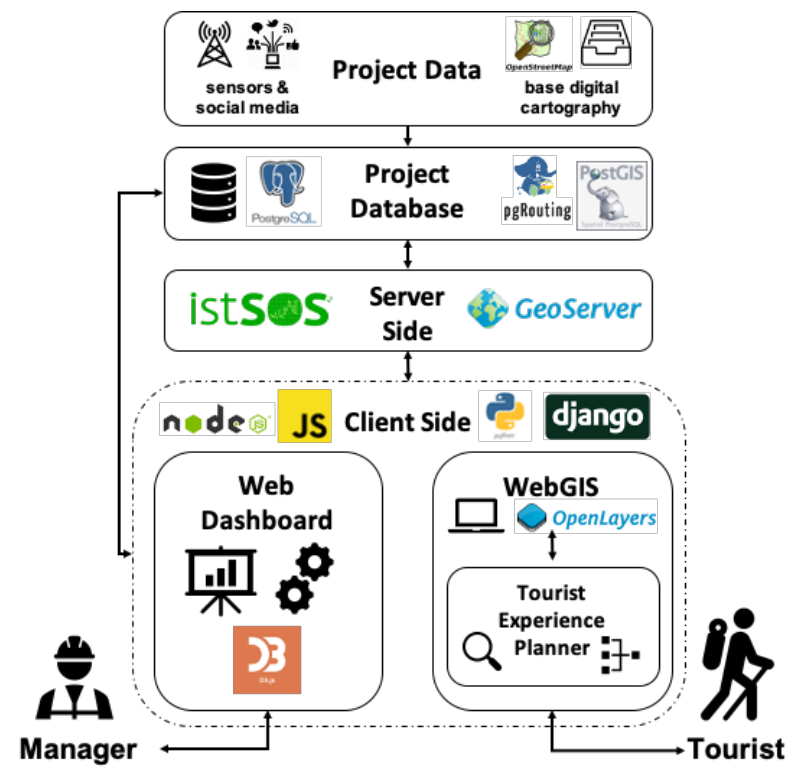

Figure 2. Schematic software architecture of the INSUBRI.PARKS IT infrastructure

A primary concern in the design of the IT infrastructure regarded the technology costs and the long term maintenance of the system. Due to the diverse technological background of the project partners, the selection of technologies for the infrastructure leverages exclusively already existing and stable FOSS and open standards for the software tools development. This choice has a dual purpose. On one end, FOSS technologies offer reliable software facilities by providing large room for customization as well as ensuring partners equitable access to the sys- tem functionalities that is not bounded by any license constraint (Brovelli et al., 2017). On the other hand, the usage of international standard favours the interoperability and the decoupling of back-end development from the front-end since the communication among components occurs according to defined and commonly accepted protocols and formats. This permits the reuse of technical items and the minimization of costs and efforts (Cannata et al., 2015). In the next section, a more detailed description on both usage and functionalities of the IT infrastructure components is included.

\section{IT INFRASTRUCTURE COMPONENTS}

\subsection{Project data}

The target data of the INSUBRI.PARKS project primarily consists of base cartographic information such as pedestrian path networks, accommodation and tourist facilities, and Points Of Interest (POIs) located in the project areas. Path networks and POIs layers are retrieved from two main sources, namely the OpenStreetMap (OSM, https://www.openstreetmap.org) the world largest open spatial database - and the partner parks archives. Target OSM layers are selectively downloaded using tags (https://wiki.openstreetmap.org/wiki/Tags). By means of manual preprocessing, OSM layers are clipped over the study areas and merged with partner archives layer in order to obtain a complete and harmonized spatial dataset, in terms of attributes, format and projection. Categories are assigned to each POIs according to the core themes of the INSUBRI.PARKS which correspond to semantic groups of attractions that are present in the project areas. These core themes are History, Military, Religious, Anthropic activity, Sport, and Nature (Oxoli et al., 2019). Concerning accommodation and tourist facilities layers, these are not included in the current version of IT infrastructure. Integration strategies are under investigation and they are focusing on the exploitation of external database by means of REpresentational State Transfer (REST) Application Programming Interface (API) services. The need for programmatic access to these particular layers relies on the fact that features such as accommodation facilities change over time likely requiring constant updates if handled as a static layer in the database. Possible sources are represented by the TripAdvisor ${ }^{\circledR}$ API (https://developer-tripadvisor.com/contentapi) and governmental open data portals, e.g. the Lombardia Open Data (https://www.dati.lombardia.it).

Besides the base cartographic information, data from the crowd such as social media posts, are considered. In particular, a Twitter crawler module, based on the Tweeter API (https://developer.twitter.com) is included in the server-side to fetch the tweets stream posted in the project areas and stored it into the database. This kind of information is intended for the virtual monitoring of the visitors' activities in the parks and neighbouring areas. This kind of information will be used to empower both on-line monitoring and off-line analysis to get insight into e.g.the most visited locations, the spatio-temporal behaviour of the visitors as well as it may support the computation of performance indicators for the project. Finally, a physical sensors network will be deployed at the principal tourist hot spots of the area. The sensors consist of smart steel platforms with embedded hardware that enables the detection of visitors passages and the convey of these observations into a database through the web. These observations, as for the social media data, will be exploited to better understand tourism fluxes features and better design both planning and marketing activities. 


\subsection{Server-side}

The server-side of the infrastructure is implemented to dynamically retrieve, organize, and expose the project data between the project database and the client applications. It is based on GeoServer and istSOS which manage respectively the cartographic information and the sensor observations through web services compliant with the OGC standards. In particular, GeoServer provides dedicated WMS and WFS and services to distribute geographic data while istSOS implements the Sensor Observation Service (SOS) OGC standard to collect and dispatch time-series observations collected by sensors.

\subsection{WebGIS}

The WebGIS client application is based on the OpenLayers library that supports WMS and WFS by implementing dynamic maps in the browser to illustrate the project areas and the available itineraries and attractions (Figure 3).

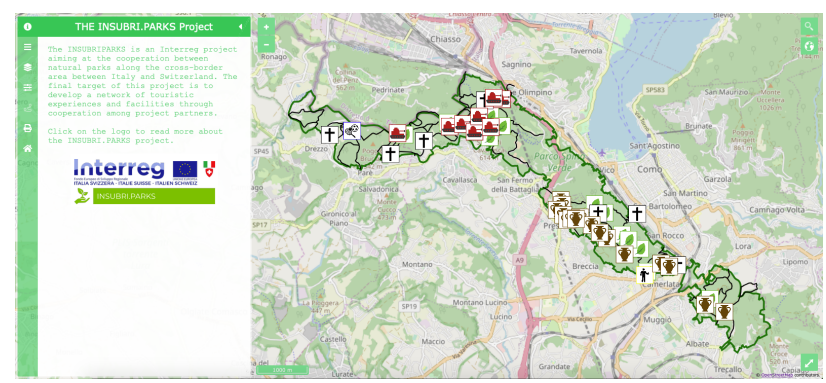

Figure 3. Prototype of the INSUBRI.PARKS WebGIS

The WebGIS is wrapped into the Python Django framework that handles the application deployment on the web. Typical operations such as browsing, querying and visualizing digital cartographic layers are enriched with a custom plugin enabling users to plan their personal experiences in the parks. The custom plugin is called Tourist Experience Planner and combines Multi-Criteria Decision Making (MCDM) and spatial analysis algorithms by enabling the computation and the rendering of the best paths to walk according to the users' expressed preferences. Computations involve the path network layers to which edges are assigned the number of POIs of the different core project themes that are reached by the edge. The interface collects dynamically the preferences from the user expressed as weight form 1 to 5 for each of the themes. Then, the user is requested to select a park area and, optionally, both an entry and exit gate of the path networks and a maximum distance to walk. These inputs are passed to a Python processing script which is run in the back-end. The script mainly exploits the Analytic Hierarchy Process (AHP) (Saaty, 2008) and the K-Shortest Paths (KSP) (Hadjiconstantinou, Christofides, 1999) algorithms to define and display on the WebGIS map the list of most suitable itineraries according to the user expressed preferences (Figure 4). The KPS computation is provided by the pgRouting extension of the PostgreSQL database while the AHP is developed by means of custom Python code. This innovative tool aims at providing an immersive virtual experience to visitors, which are allowed to interact with their data of interest improving their connection and involvement with the INSUBRI.PARKS.

A prototype of the INSUBRI.PARKS WebGIS has been published at the following link: http://insubriparks.como.polimi.it:8000/webgis.

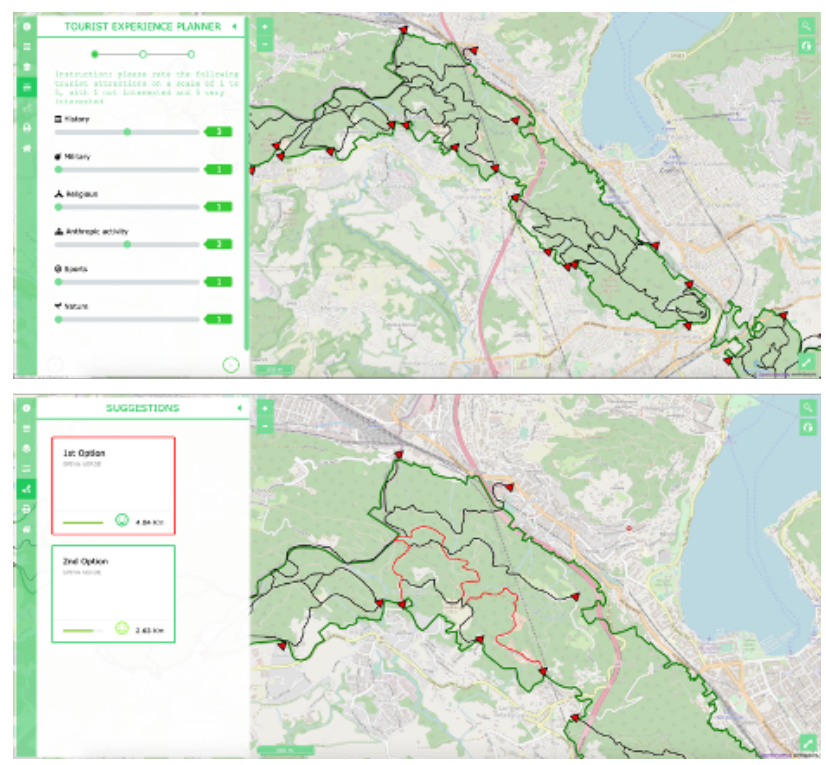

Figure 4. Tourist Experience Planner plugin included in the WebGIS of INSUBRI.PARKS: User's preferences collection tab (top), and results rendering (down)

\section{4 dashboard}

Regarding the use of social media and sensors data, a web dashboard is created to provide a standardized access point to this information. The dashboard is conceived to allow only park managers and their collaborators handling the data which may include content that cannot be made public due to data licences or privacy issues. This is the case e.g. of Twitter which granted data access exclusively for internal use to the INSUBRI.PARKS project.

The web dashboard is mainly based on the Python Django web framework and the D3.js library which provide the system with both user authentication functionalities and interactions with data that are retrieved directly from the database of the infrastructure. Interaction is enabled by means of dynamic graphs and maps enriched with filtering widgets allowing to explore data both in space and time (Figure 5). Indeed, target data of the dashboard are mainly time series of geolocated observations that are intended to support a better understanding and characterization of the tourism fluxes within the project area. The main purpose is to develop metrics to quantify these fluxes which are expected to increase and/or better distribute across the study areas as an effect of the project actions. To that end, analytical widgets to allow extracting indicators and summary statistics from the data will be included to facilitate project impacts assessment both during its execution and in the long term.
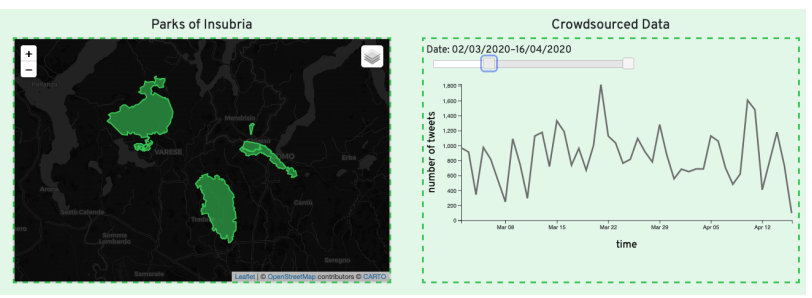

Figure 5. Prototype interface of the INSUBRI.PARKS web dashboard 


\section{CONCLUSIONS AND FUTURE WORK}

We presented here the work carried out for the INSUBRI.PARKS IT infrastructure development. At the time that this paper is written, the INSUBRI.PARKS project is at onethird of its nominal duration (July 2019 - June 2022). The presented IT components have to be intended as intermediate outcomes which might be partially re-designed or adjusted along with the extensive testing phases which have been planned.

Despite the early stage of the IT components, the developed tools promise to empower the INSUBRI.PARKS partners with new digital assets that can be directly spent in the marketing operations as well as in the monitoring of the project areas. These benefits coupled with the provision of new tourism facilities, as well as the extension of the tourism offer, outline as the main tangible outcome from the visitors' perspective. On the other hand, the availability of ordered and accessible records and maps through the IT infrastructure may benefit also the monitoring of project progresses and impacts. This aims at measuring the actual changes in the tourism fluxes of which growth represents the main goal of the project. To that end, both real-time and off-line analysis e.g. of sensor and social media data are here considered as critical to the assessment of performances of the tourism system and the provision of valuable insight to the managers of the parks. Finally, the role of FOSS and GIS technologies in the development of the INSUBRI.PARKS IT infrastructure was spelt out according to both the technical and the economic perspective.

Additional tools and data for the IT infrastructure are being considered. Among auxiliary data sources, we mentioned the inclusion of tourism accommodations layers into the WebGIS by means of REST API. Concerning tools, the adoption of mobile solutions for field data collection is under investigation to allow visitors and park rangers contributing to the reporting of parks status and the reviewing of parks itineraries and attractions. The above tasks will be tackled within the future development of the INSUBRI.PARKS project.

\section{ACKNOWLEDGEMENTS}

This work has been funded by the project "INSUBRI.PARKS" within the Interreg Co-operation Programme 2014 - 2020 (ID 605472). The authors would like to acknowledge Candan Eylül Kilsedar and Matteo Rizzi for the support provided in the development of the IT tool prototypes.

\section{REFERENCES}

Antonovic, M., Brovelli, M., Cannata, M., Cardoso, M., Kilsedar, C., Minghini, M., Zamboni, G., 2015. Promoting slow tourism through foss $4 \mathrm{~g}$ web mapping: an italian-swiss case study. Geomatics Workbooks 12 - FOSS4G Europe 2015, 99104

Brovelli, M. A., Minghini, M., Moreno-Sanchez, R., Oliveira, R., 2017. Free and open source software for geospatial applications (FOSS4G) to support Future Earth. International journal of digital earth, 10(4), 386-404.

Buhalis, D., 2003. eTourism: Information technology for strategic tourism management. Pearson education.
Buhalis, D., Amaranggana, A., 2015. Smart tourism destinations enhancing tourism experience through personalisation of services. Information and communication technologies in tourism 2015, Springer, 377-389.

Cannata, M., Colombo, M., Antonovic, M., Cardoso, M., Delucchi, A., Gianocca, G., Brovelli, M. A., 2015. ” i cammini della regina"-open source based tools for preserving and culturally exploring historical traffic routes. EGU General Assembly Conference Abstracts, 17.

Chon, K. S., 2013. Geography and tourism marketing. Routledge.

Gretzel, U., Sigala, M., Xiang, Z., Koo, C., 2015. Smart tourism: foundations and developments. Electronic Markets, 25(3), 179-188.

Hadjiconstantinou, E., Christofides, N., 1999. An efficient implementation of an algorithm for finding $\mathrm{K}$ shortest simple paths. Networks: An International Journal, 34(2), 88-101.

Jovanović, V., 2016. The application of GIS and its components in tourism. Yugoslav Journal of Operations Research, 18(2).

Li, Y., Hu, C., Huang, C., Duan, L., 2017. The concept of smart tourism in the context of tourism information services. Tourism Management, 58, 293-300.

Masron, T., Ismail, N., Marzuki, A., 2016. The conceptual design and application of web-based tourism decision support systems. Theoretical \& Empirical Researches in Urban Management, 11(2).

Oxoli, D., Cannata, M., Terza, V., Brovelli, M., Verde, P. R. S., 2019. NATURAL HERITAGE MANAGEMENT AND PROMOTION THROUGH FREE AND OPEN SOURCE SOFTWARE: A PRELIMINARY SYSTEM DESIGN FOR THE INSUBRIPARKS PROJECT. International Archives of the Photogrammetry, Remote Sensing and Spatial Information Sciences, 42(4/W14).

Prell, C., Hubacek, K., Reed, M., 2009. Stakeholder analysis and social network analysis in natural resource management. Society and Natural Resources, 22(6), 501-518.

Saaty, T. L., 2008. Decision making with the analytic hierarchy process. International journal of services sciences, 1(1), 83-98.

Wei, W., 2012. Research on the application of geographic information system in tourism management. Procedia Environmental Sciences, 12, 1104-1109. 